\title{
Dark lesion elimination based on area, eccentricity and extent features for supporting haemorrhages detection
}

\author{
Vesi Yulyanti ${ }^{a}$, Hanung Adi Nugroho, ${ }^{a,}$, Igi Ardiyanto ${ }^{a}$, Widhia KZ Oktoeberza ${ }^{b}$ \\ ${ }^{a}$ Department of Electrical and Information Engineering, Faculty of Engineering, Universitas Gadjah Mada, Yogyakarta 55281, Indonesia \\ ${ }^{b}$ Department of Informatics, Faculty of Engineering, Universitas Bengkulu, Bengkulu 38371, Indonesia
}

Article history:

Received: 22 April 2019 / Received in revised form: 25 May 2019 / Accepted: 27 May 2019

\begin{abstract}
One of the complications due to the long-term of diabetes is retinal vessels damaging called diabetic retinopathy. It is characterised by appearing the bleeding spots in the large size (haemorrhages) on the surface of retina. Early detection of haemorrhages is needed for preventing the worst effect which leads to vision loss. This study aims to detect haemorrhages by eliminating other dark lesion objects that have similar characteristics with haemorrhages based on three features, i.e. area, eccentricity and extent features. This study uses 43 retinal fundus images taken from DIARETDB1 database. Based on the validation process, the average level of sensitivity gained is $80.5 \%$. These results indicate that the proposed method is quite capable of detecting haemorrhages which appear in the retinal surface.
\end{abstract}

Keywords: Retinal image; haemorrhages detection; dark lesions; eccentricity feature, extent feature.

\section{Introduction}

Diabetes is one of the major health problems in developing countries. World Health Organisation (WHO) has reported that around 171 million people lived with diabetes in 2000 and it is predicted will be increased to 366 million diagnosed diabetics worldwide by 2030. Moreover, International Diabetes Federation (IDF) has predicted that more than 400 million people by 2030 will be threatened by glucose disorders.

Diabetes is classified into two types, i.e. type-1 and type-2. When the pancreas merely capable to produce insulin in slightly, it is called type- 1 diabetes, while type- 2 diabetes is commonly caused by unhealthy life such as obesity [1]. One of the impacts of diabetes is diabetic retinopathy which damaging the retinal vessels and causing of blindness $[1,2]$.

The bleeding spots will appear in the surface of retina in small size (microaneurysms) and the large one (haemorrhages). Sometimes, these bleeding spots disappear by themselves. However, without treatment immediately, it often recurs and gets worst. Therefore, early detection and diagnose are needed in order to reduce the eye damage and prevent the blindness [3].

Some research works related to haemorrhages detection for early diagnosing of diabetic retinopathy have been conducted. Bharaly, et al. detected haemorrhages based on thresholding algorithm on the green channel. Contrast limited adaptive histogram equalisation (CLAHE) was applied in the preprocessing step followed by median and mean filtering. Retinal vessels was detected by using region growing method and then eliminated from the image to obtain final area of haemorrhages [4].

Mane, et al. used matched filter algorithm which has modified for detecting retinal vessels. A total of 11 features were extracted from filtered image. These features include area, aspect ratio, circumference, eccentricity, average intensity, standard deviation, the length of main axis, the length of minor axis, density, equivalent diameter and roundness. Afterwards, these extracted features were used as input in the classification process based on support vector machine (SVM) classifier. This approach was tested on 89 retinal images from DIARETDB1 database. The classification results achieved the accuracy, sensitivity and specificity of $96.62 \%, 96.42 \%$ and $100 \%$, respectively [5].

Sreng, et al. [6] extracted the reddish dark lesions based on some features to facilitate haemorrhages detection. Foveal area was extracted based on density feature, while that of retinal vessels based on eccentricity feature. Microaneurysms which has similar characteristics with haemorrhages was extracted based on area feature. This study was conducted on 579 fundus images taken from Bhumibol Adulyadej Hospital. The accuracy achieved is $90 \%$ with the processing time of 6.23 seconds/ image.

To complete the study of haemorrhages detection, this paper proposes an approach to detect haemorrhages by eliminating dark lesion objects based on area, eccentricity and extent feature. The structure of this paper is organised as follows. Section II explains materials and method used. The results and discussion are presented in Section III followed by conclusion in Section IV. 


\section{Materials and Methods}

A number of 43 retinal fundus images taken from DIARETDB1 were used in this study [7]. These images are available in RGB (*.png) format with the resolution of 1500x1152 pixels. The images were captured by using digital fundus camera from an angle of 500 field-of-view with a variety of settings (flash intensity, shutter speed, aperture, gain) controlled by the system. Moreover, this database also provides the ground truth images for facilitating the validation at the end process. Therefore, this database was chosen to be processed in this study. The sample of original and ground truth images are shown in Fig. 1.
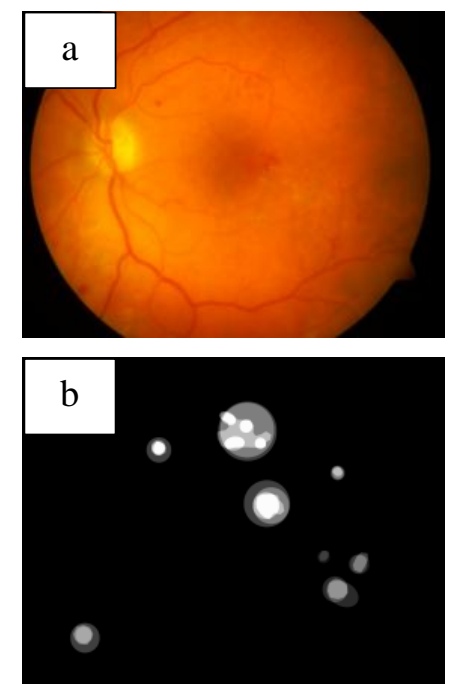

Fig. 1. The examples of: (a) RGB original image; (b) ground truth image
This study proposed two schemes process, namely haemorrhages detection and retinal vessels detection as depicted in the Fig. 2. The original image was getting some ways in pre-processing step. After that, pre-processed image undergoes parallel process for detecting the candidates of haemorrhage and retinal vessels as well. By involving subtraction operation, final haemorrhages were detected.

\subsection{Pre-processing}

Firstly, the retinal image was resized to $0.6 \mathrm{x}$ of the original size or equal to $900 \times 692$ pixels. Afterwards, channels extraction was conducted to separate green channels from the two others channel. Green channel was chosen since it has the best contrast than others as described in Fig. 3.

Then, extracted green channel was processed by contrast limited adaptive histogram equalisation (CLAHE) followed by contrast adjustment for improving the contrast image and make the target object more clearly. The results of preprocessing step are shown in Fig. 4. From the adjusted image, we can see that retinal vessels and haemorrhages tend to be equally prominent. So, before step across to the next step, the enhanced image was applied by median filtering. Two scales of median filtering used are $s_{1}=10$ and $s_{2}=35$ (Fig. 5). It was conducted in parallel process. The chosen of two scales is based on the experiment result with several different scales.

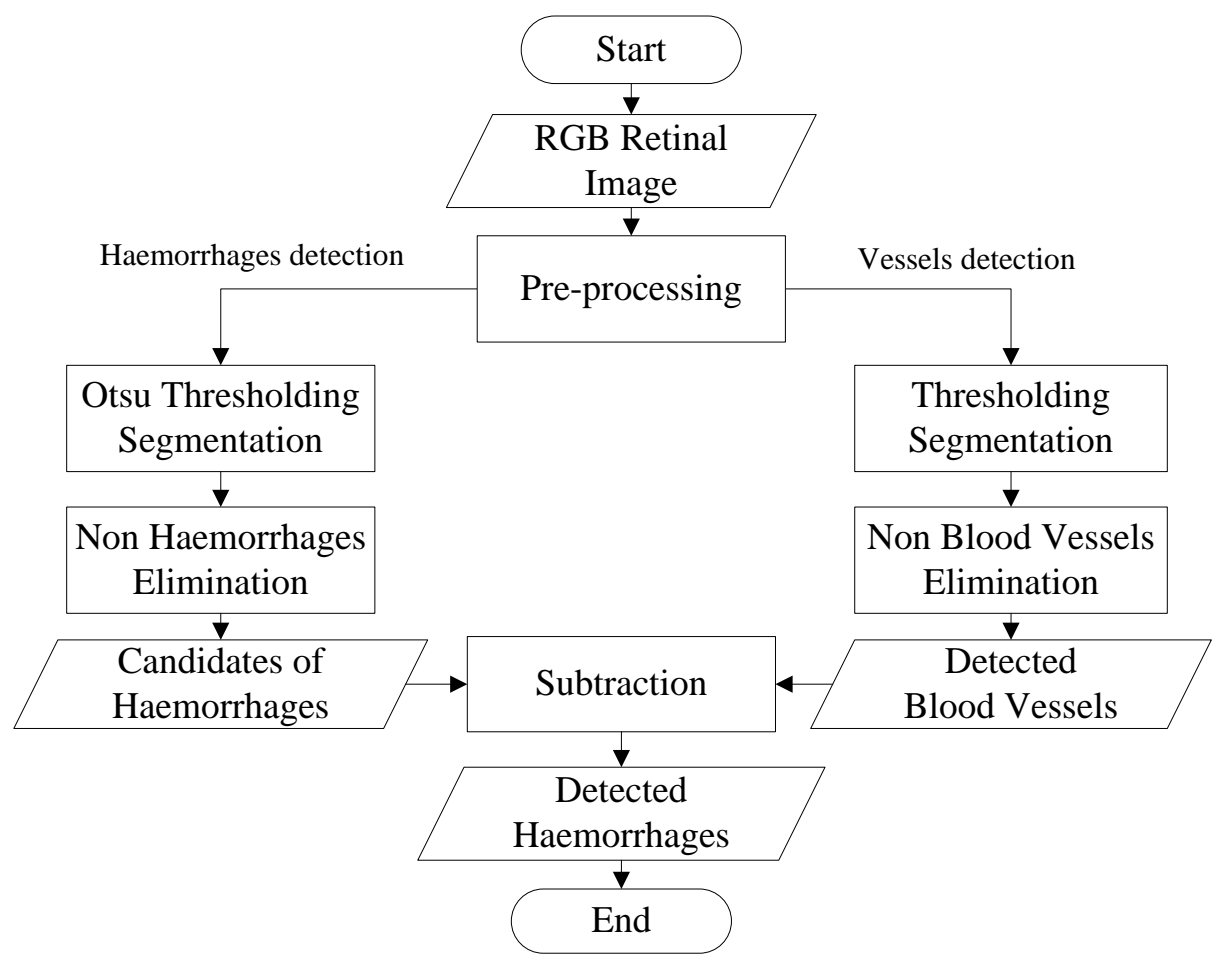

Fig. 2. Flowchart of the proposed approach 

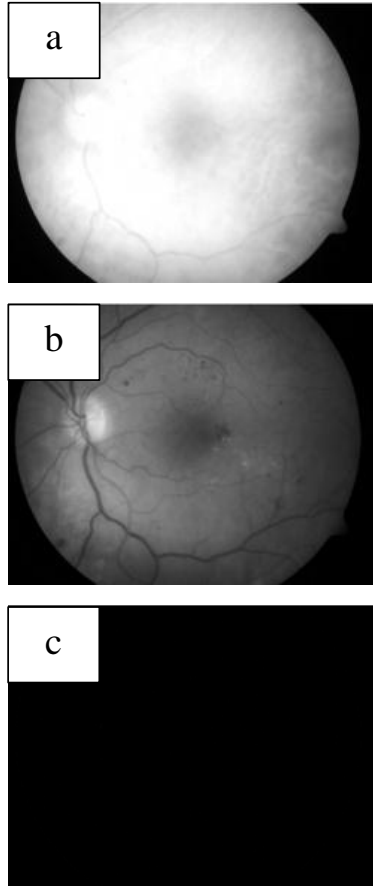

Fig. 3 The images results of channel extraction: (a) red;

(b) green; and (c) blue channels
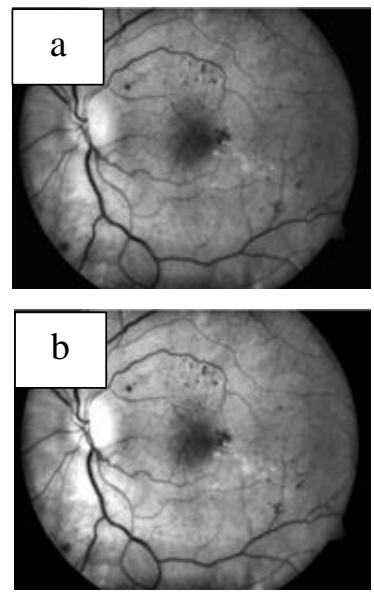

Fig. 4. The results of pre-processing: (a) CLAHE; (b) contrast adjustment
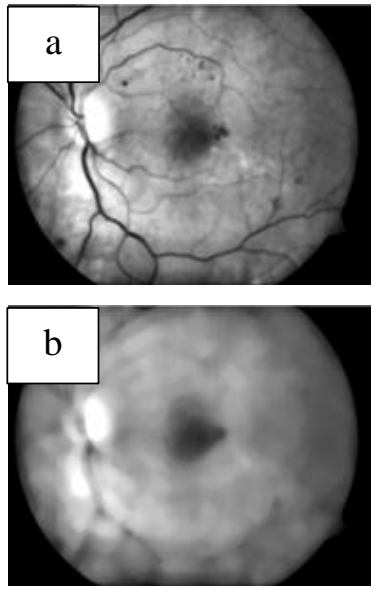

Fig. 5. The results of median filtering: (a) $s 1=10$; (b) $s 2=35$
Next, the adjusted and filtered images were denoted by $I_{A}$ and $I_{B}$, respectively. Here, we intended by subtracting $I_{A}$ from $I_{B}$ (1) to make the dark area such as retinal vessels, microaneurysms and haemorrhages be brighter than background area. Moreover, we also can see that the fovea area is removed from the image as presented in Fig. 6.

$$
I_{\text {Sub }}=I_{B}-I_{A}
$$

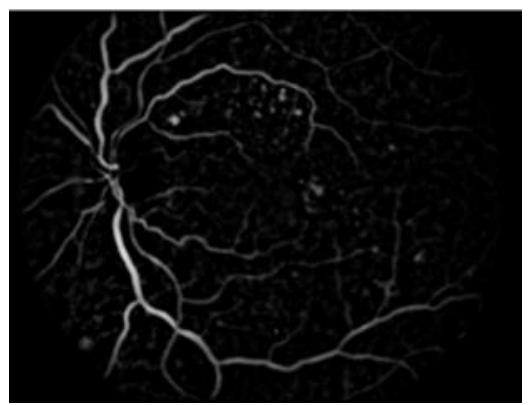

Fig. 6. The image result of subtraction process $\left(I_{S u b}\right)$

\subsection{Haemorrhages Detection}

The image result of subtraction process was then applied by Otsu thresholding in order to over separate the object and the background area. As shown in Fig. 7 (a), there are still contain retinal vessels, microaneurysms and others object that having similar character as dark areas. So, to eliminate these unwanted objects (non-haemorrhages), three features namely area, eccentricity and extent were involved in this process because these features represent the shape of object.

In shape, haemorrhages have the difference from the retinal vessels. While in size, haemorrhages are bigger than microaneurysms. If the object area is less than $50 \mu \mathrm{m}$, it was eliminated due to denoted as noises or microaneurysms. The area that should be eliminated ones is the object having the eccentricity value approaches to 1 , since it was denoted as line objects or retinal vessels. Also, the object with the extent value approaches to 0 (zero), it was eliminated and denoted as retinal vessels as well. So, the candidates of haemorrhages were obtained as shown in Fig. 7 (b).
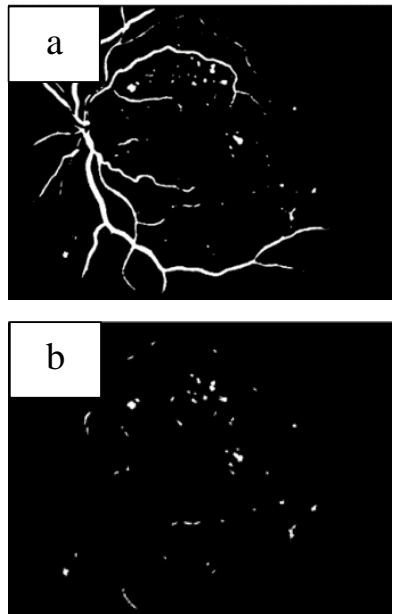

Fig. 7. The image results of

(a) Otsu thresholding; (b) candidates of haemorrhages 


\subsection{Retinal Vessels Detection}

Although the retinal vessels had eliminated from the image, there are still left small pieces of retinal vessels as shown in Fig. 7 (b). Therefore, it is important to detect the whole area of the retinal vessels and then remove them for getting the final area of haemorrhages. Here, we used the image result of subtraction process $\left(I_{S u b}\right)$ to be applied by thresholding method with the threshold value of 0.04 .

Afterwards, other objects (non-retinal vessels) detected were eliminated by considering the eccentricity and extent features. The objects with eccentricity value more than 0.95 and extent value less than 0.3 will be eliminated from the image. The result of retinal vessels detection is depicted in Fig. 8. Lastly, to obtain the final area of haemorrhages, subtraction process was applied between candidates haemorrhages (Fig. 7 (b)) to the detected retinal vessels (Fig. 8).

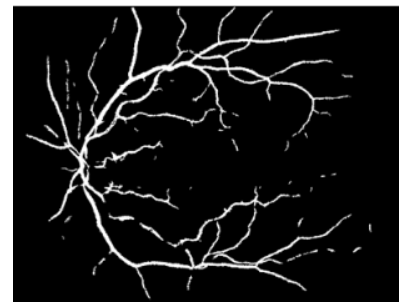

Fig. 8. The result of retinal vessels detection

\subsection{Validation Process}

To find out the performance of the proposed method, we calculated the sensitivity value by using (2). This formulation involves two statistical parameters, namely true positive (TP) and false negative (FN).

$$
\text { Sensitivity }=\frac{T P}{T P+F N} \times 100 \%
$$

\section{Results and Discussion}

Having obtained the images result of two schemes proposed, i.e. the candidate area of haemorrhages and detected retinal vessels, the next process to do is subtracting both images for gaining the final result of haemorrhages detection. Here, there are no more retinal vessels, microaneurysms and others unwanted object as shown in Fig. 9 (a). However, haemorrhages which coincide the retinal vessels will be eliminated while the subtracting process (see Fig. 9 (b)).

Final haemorrhages obtained were then validated to the ground truth image (Fig. 9 (c)) which labelled by several experts. This process was done in order to ascertain the truth of haemorrhages detected. Since the labelled haemorrhages in the ground truth image is not a definite position (merely the area which is estimated containing haemorrhages), so each detected haemorrhages is in labelled area in the ground truth image, it is defined as true positive (TP). False negative (FN) defines when there is no detected haemorrhages in the labelled area. Validation process is illustrated in Fig. 9 (d).
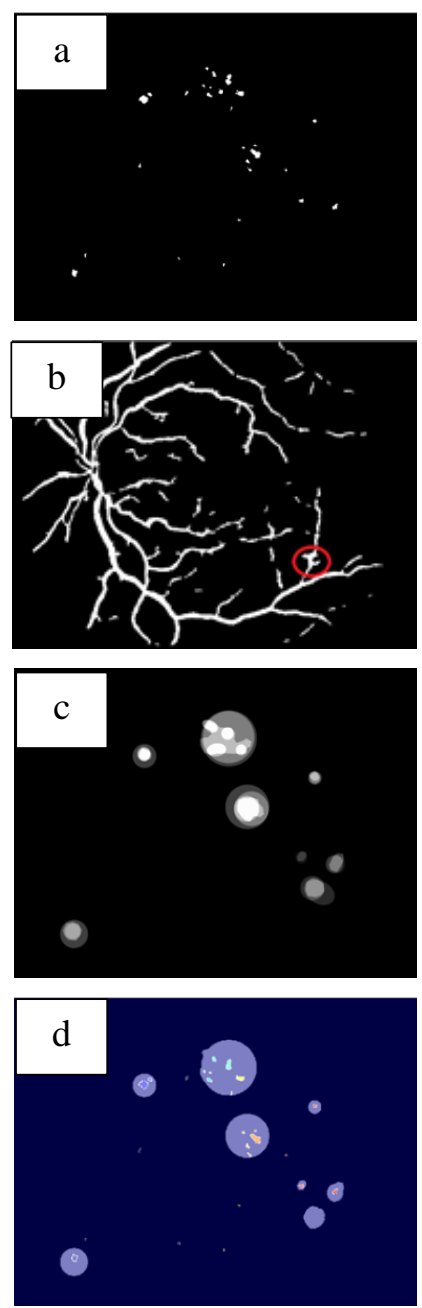

Fig. 9. (a) Final detected haemorrhages; (b) Haemorrhages coincide with retinal vessels; (c) ground truth image; (d) validation process

Having gained the value of TP, and FN, the next process conducted is calculated the sensitivity value. The results of sensitivity are presented in Fig. 10. As shown in Fig. 10, we can see that the highest sensitivity value is $100.0 \%$, while the lowest one is $16.7 \%$. From the whole number of images, the average value of sensitivity achieved is $80.5 \%$. 


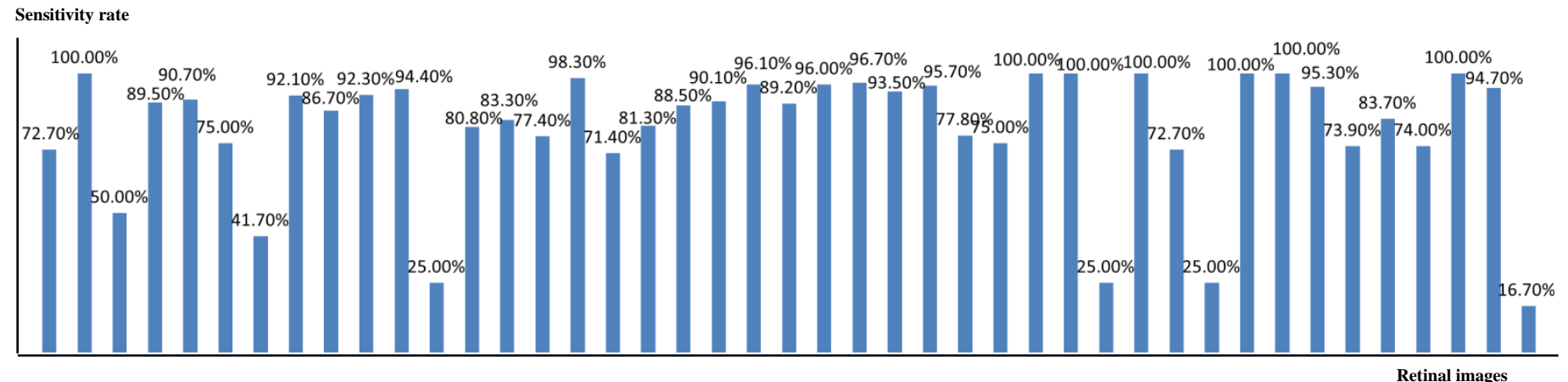

Fig. 10. The results of sensitivity

\section{Conclusion}

An approach for detecting haemorrhages in retinal fundus image has been proposed. Three features, i.e. area, eccentricity and extent features are involved in order obtaining the final result of haemorrhages detection. In this study, the average value of sensitivity gained is $80.5 \%$. This result indicates that the proposed method is able to detect the presence of haemorrhages. However, it still needs to be improved due to the percentage results are not maximized yet. There are several areas of detected haemorrhages lost due to coinciding with retinal vessels, which have also an impact on the validation results.

\section{Acknowledgements}

This study is funded by Directorate General of Higher Education, Ministry of Research, Technology and Higher Education, Republic of Indonesia. We would also like to thank the Intelligent Systems research group members in our Department for sharing and discussing.

\section{References}

1. K. G. M. M. Alberti and P. F. Zimmet, Definition, diagnosis and classification of diabetes mellitus and its complications. Part 1: diagnosis and classification of diabetes mellitus. Provisional report of a WHO consultation, Diabetic medicine. 15 (1998) 539-553.

2. R. Goldenberg and Z. Punthakee, Definition, classification and diagnosis of diabetes, prediabetes and metabolic syndrome, Canadian journal of diabetes. 37 (2013) S8-S11.

3. S. Kanth, A. Jaiswal, and M. Kakkar, Identification of different stages of Diabetic Retinopathy using artificial neural network, Sixth International Conference on Contemporary Computing (IC3), 2013, pp. 479-484.

4. P. Bharali, J. P. Medhi, and S. Nirmala, Detection of hemorrhages in diabetic retinopathy analysis using color fundus images, 2015 IEEE 2nd International Conference on Recent Trends in Information Systems (ReTIS), 2015, pp. 237-242.

5. V. M. Mane, R. B. Kawadiwale, and D. Jadhav, Detection of red lesions in diabetic retinopathy affected fundus images, 2015 IEEE International Advance Computing Conference (IACC), 2015, pp. 56-60.

6. S. Sreng, N. Maneerat, D. Isarakorn, K. Hamamoto, and R. Panjaphongse, Automatic hemorrhages detection based on fundus images, 2015 7th International Conference on Information Technology and Electrical Engineering (ICITEE), 2015, pp. 253-257.

7. V. K. Tomi Kauppi, J.-K. Kamarainen, L. Lensu, I. Sorri, A. Raninen, R. Voutilainen, J. Pietilä, H. Kälviäinen, and H. Uusitalo, DIARETDB1 Standard Diabetic Retinopathy Database Calibration level 1, ed, 2007. 